\title{
Electronic excitation spectrum of thiophene studied by symmetry- adapted cluster configuration interaction method
}

AUTHOR(S):

Wan, J; Hada, M; Ehara, M; Nakatsuji, H

\section{CITATION:}

Wan, J ...[et al]. Electronic excitation spectrum of thiophene studied by symmetryadapted cluster configuration interaction method. JOURNAL OF CHEMICAL PHYSICS 2001, 114(2): 842-850

ISSUE DATE:

2001-01-08

URL:

http://hdl.handle.net/2433/50181

\section{RIGHT:}

Copyright 2001 American Institute of Physics. This article may be downloaded for personal use only. Any other use requires prior permission of the author and the American Institute of Physics. 


\title{
Electronic excitation spectrum of thiophene studied by symmetry-adapted cluster configuration interaction method
}

\author{
Jian Wan, Masahiko Hada, Masahiro Ehara, and Hiroshi Nakatsujia) \\ Department of Synthetic Chemistry and Biological Chemistry, Graduate School of Engineering, \\ Kyoto University, Sakyo-ku, Kyoto 606-8501, Japan
}

(Received 27 June 2000; accepted 23 October 2000)

\begin{abstract}
Electronic excitation spectrum of thiophene was investigated by the symmetry-adapted cluster (SAC)/SAC configuration interaction method. Seventy singlet and four lowest triplet electronic states of thiophene were computed to give a detailed satisfactory theoretical interpretation of the vacuum ultraviolet (VUV) spectrum and the electron energy loss spectrum of thiophene. The present calculations gave the $2{ }^{1} A_{1}$ valence state at $5.41 \mathrm{eV}$ and the $1{ }^{1} B_{2}$ valence state at $5.72 \mathrm{eV}$ with oscillator strengths 0.0911 and 0.1131 , respectively, and the $5{ }^{1} A_{1}$ valence state at $7.32 \mathrm{eV}$ and the $4^{1} B_{2}$ valence state at $7.40 \mathrm{eV}$ with oscillator strengths 0.3614 and 0.1204 , respectively. These valence-excited states were assigned to the two strong absorption bands of the VUV spectrum centered around 5.5 and $7.05 \mathrm{eV}$, respectively. A number of Rydberg transitions were obtained and assigned to the $6.0,6.6$, and 7.5-8.7 eV, etc. energy regions. The similarities and differences in the electronic excitations between thiophene and other five-membered ring compounds were discussed. The accuracy and assignment of the present results are compared with those of the recent theoretical studies by CASPT2 and multireference double configuration interaction methods. (C) 2001 American Institute of Physics.
\end{abstract}

[DOI: 10.1063/1.1332118]

\section{INTRODUCTION}

The electronic spectra of the five-membered ring compounds cyclopentadiene (CP), furan, and pyrrole have been the subjects of many experimental and theoretical studies. ${ }^{1-37}$ The interest in these molecules is not surprising, considering that these molecules are fundamental units in many important biological molecules. Furthermore, their electronic excitation spectra have received much attention in recent years as benchmark examples for theoretical studies of excited states. The electronic excited states of $\mathrm{CP}$, furan, and pyrrole have been successfully studied in our recent studies $^{38,39}$ using the symmetry-adapted cluster configuration interaction (SAC-CI) method that offers a consistent interpretation of the electronic excitation spectra of these molecules. As a series of this study, we examine here the excitation spectrum of thiophene. By a replacement of oxygen with sulfur, a second-row element, very interesting changes occur in both ground and excited states.

The electronic absorption spectrum of thiophene has been intensively investigated since the beginning of the last century. A comparison of condensed phase spectra ${ }^{12-14}$ with gas phase data confirms the valence nature of the strong bands around 5.5 and $7.05 \mathrm{eV}$ and suggests that the weak fine structure with onset around $6.0 \mathrm{eV}$ is attributed to a Rydberg excited state. The magnetic circular dichroism (MCD) spectrum of thiophene ${ }^{16-18}$ in solution of hexane shows two bands at 5.27 and $5.64 \mathrm{eV}$, respectively, having opposite signs in their $B$-values. Electron energy loss (EEL)

\footnotetext{
a) Author to whom correspondence should be addressed. Electronic mail: hiroshi@sbchem.kyoto-u.ac.jp
}

spectra have been reported, ${ }^{19-21}$ and they show that two lowest-lying triplet excited states (both ${ }^{3} \pi-\pi^{*}$ ) were located at about 3.8 and $4.7 \mathrm{eV}$. These experimental data will be discussed in detail below in the light of the present theoretical calculations.

To the best of our knowledge, there have been two sophisticated $a b$ initio studies on the excited states of thiophene. Bendazzoli et al. ${ }^{12}$ obtained six lowest excited $A_{1}$ states (three for each of singlet and triplet), and eight lowest of each of the symmetries $A_{2}, B_{2}$, and $B_{1}$ by the configuration interaction (CI) method using a double-zeta basis set. In their study, two pairs of valence $\pi-\pi^{*}$ excited states were given in the order, ${ }^{1} A_{1}$ (middle) $<{ }^{1} B_{2}$ (middle) $<{ }^{1} A_{1}$ (strong) $<{ }^{1} B_{2}$ (very strong). More extensive $a b$ initio study was given by the CASPT2 (Ref. 29) method giving the same intensity order as Bendazzoli's, but only the $1 a_{2} \rightarrow n$ $=3$ and $3 b_{1} \rightarrow n=3$ series of Rydberg states in the lower energy region were examined in the CASPT2 studies. Recently, Palmer et al..$^{32}$ reported a multireference double configuration interaction MRD-CI study, in which the intensity order of the valence $\pi-\pi^{*}$ excited states was ${ }^{1} A_{1}$ (middle) $<{ }^{1} B_{2}$ (middle) $<{ }^{1} A_{1}$ (very strong) $<{ }^{1} B_{2}$ (strong). The order of these four excited states was the same in three bases (DZPR, TZVP, and QZVP), however, the oscillator strengths were different even qualitatively. In the latest experimental vacuum ultraviolet (VUV) spectroscopic study, a number of Rydberg transitions in the energy region of 6.0, 6.6, and 7.7-8.8 eV were observed, though they were discussed only briefly since the MRD-CI energies were too high to make a theoretical assignment, especially in the higher energy region. There are still some critical inconsistencies between the 


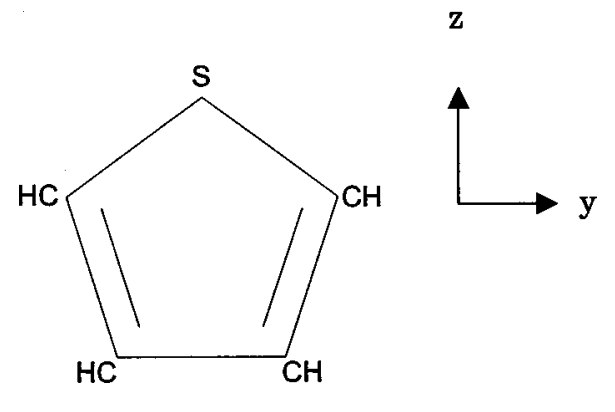

FIG. 1. Thiophene.

results of these two high-level ab initio studies. In particular, the calculated excitation energies and oscillator strengths for the second pair of valence $\pi-\pi^{*}$ excited states $\left({ }^{1} A_{1}\right.$ and ${ }^{1} B_{2}$, corresponding to the most intense band in the VUV spectrum) were quite different between the CASPT2 and MRD-CI studies.

In this article, we study the electronic excitation spectrum of thiophene by the SAC-CI method as a series of our recent studies ${ }^{38,39}$ on the five-membered ring compounds. There are two objectives in the present study: one is to reduce ambiguity in the assignment of the electronic spectrum of thiophene in a wide energy range, and the other is to give a theoretical basis for further experimental studies on thiophene. In Sec. II we outline the computational details. Calculated results and discussions are presented in Sec. III. A summary is given in Sec. IV.

\section{COMPUTATIONAL DETAILS}

All the calculations have been performed at the experimentally determined ground state geometry ${ }^{40}$ with $C_{2 v}$ symmetry. The molecular plane used is on the $y z$-plane, and the $C_{2}$ axis on the $z$-axis as illustrated in Fig. 1. The calculated excitation energies are vertical in nature.

Dunning's augmented correlation consistent valence triple-zeta (AUG-cc-pVTZ) ${ }^{41}$ basis set was used for S and C atoms though one $f$-function was removed from each $\mathrm{C}$ atom, and cc-pVTZ ${ }^{42}$ was used for $\mathrm{H}$ atoms. Additionally, a set of diffuse Rydberg functions $(5 s 5 p 5 d)$ selected from the studies of Kaufmann et al. ${ }^{43}$ was placed on the molecular center of the gravity to investigate the Rydberg states in a wide energy region. The total number of basis functions is 307 . This type of augmented basis sets was used in the studies of Christiansen et al. ${ }^{36,37}$ and in our recent calculations. ${ }^{38,39}$ This set could describe widely different electronic structures of these five-membered ring compounds in the ground state, singlet, and triplet valence and Rydberg excited states with a high accuracy. The number of Hartree-Fock canonical orbitals, calculated by the GAUSSIAN 98 package,${ }^{44}$ is 307 , consisting of 22 occupied and 285 virtual orbitals. The selfconsistent field (SCF) occupied valence orbitals and some important virtual orbitals are shown in Table I, together with their orbital energies, symmetries, and characters.

The details of the SAC/SAC-CI theory for calculating ground and excited states of molecules have been presented elsewhere, ${ }^{45-51}$ and the calculations have been performed with the local module ${ }^{51}$ of our laboratory. In all of the
TABLE I. Some important SCF occupied and unoccupied molecular orbitals (MOs).

\begin{tabular}{|c|c|c|c|}
\hline MOs & Symmetry & $\begin{array}{l}\text { Orbital energy } \\
(\mathrm{eV})\end{array}$ & Nature \\
\hline \multicolumn{4}{|l|}{ Occupied } \\
\hline 19 & $2 b_{1}$ & -14.249 & $\pi$ \\
\hline 20 & $6 b_{2}$ & -12.926 & $n(\sigma$ lone-pair orbital $)$ \\
\hline 21 (next HOMO) & $3 b_{1}$ & -9.413 & $\pi_{1}$ \\
\hline 22 (HOMO) & $1 a_{2}$ & -8.916 & $\pi_{2}($ node at sulfur $)$ \\
\hline \multicolumn{4}{|l|}{ Unoccupied } \\
\hline 23 (LUMO) & $a_{1}$ & 0.034 & $s$-Rydberg \\
\hline 24 & $b_{2}$ & 0.094 & $p_{y}$-Rydberg \\
\hline 25 & $a_{1}$ & 0.097 & $p_{z}$-Rydberg \\
\hline 26 & $b_{1}$ & 0.102 & $p_{z}$-Rydberg \\
\hline 27 & $a_{1}$ & 0.148 & $s$-Rydberg \\
\hline 28 & $a_{1}$ & 0.204 & $d_{0}$-Rydberg \\
\hline 29 & $b_{2}$ & 0.204 & $d_{-1}$-Rydberg \\
\hline 30 & $a_{2}$ & 0.210 & $d_{-2}$-Rydberg \\
\hline 31 & $b_{1}$ & 0.212 & $d_{+1}$-Rydberg \\
\hline 32 & $a_{1}$ & 0.212 & $d_{+2}$-Rydberg \\
\hline 33 & $b_{2}$ & 0.304 & $p_{y}$-Rydberg \\
\hline 34 & $a_{1}$ & 0.313 & $p_{z}$-Rydberg \\
\hline 35 & $b_{1}$ & 0.327 & $p_{x}$-Rydberg \\
\hline 36 & $a_{1}$ & 0.380 & $s$-Rydberg \\
\hline 37 & $b_{2}$ & 0.552 & $d_{-1}$-Rydberg \\
\hline 38 & $a_{1}$ & 0.553 & $d_{0}$-Rydberg \\
\hline 39 & $a_{2}$ & 0.571 & $d_{-2}$-Rydberg \\
\hline 40 & $a_{1}$ & 0.577 & $d_{+2}$-Rydberg \\
\hline 41 & $b_{1}$ & 0.578 & $d_{+1}$-Rydberg \\
\hline 42 & $b_{2}$ & 0.701 & $p_{y}$-Rydberg \\
\hline 43 & $a_{1}$ & 0.724 & $p_{z}$-Rydberg \\
\hline 44 & $b_{1}$ & 0.746 & $p_{x}$-Rydberg \\
\hline 45 & $a_{1}$ & 0.831 & $s$-Rydberg \\
\hline 46 & $b_{2}$ & 1.152 & $d_{-1}$-Rydberg \\
\hline 47 & $a_{1}$ & 1.162 & $d_{0}$-Rydberg \\
\hline 48 & $a_{2}$ & 1.214 & $d_{-2}$-Rydberg \\
\hline 49 & $a_{1}$ & 1.217 & $d_{+2}$-Rydberg \\
\hline 50 & $b_{1}$ & 1.230 & $d_{+1}$-Rydberg \\
\hline 51 & $b_{2}$ & 1.432 & $p_{y}$-Rydberg \\
\hline 52 & $a_{1}$ & 1.492 & $p_{z}$-Rydberg \\
\hline 53 & $b_{1}$ & 1.515 & $p_{x}$-Rydberg \\
\hline 54 & $a_{1}$ & 1.781 & $s$-Rydberg \\
\hline 55 & $b_{2}$ & 2.172 & $d_{-1}$-Rydberg \\
\hline 56 & $a_{1}$ & 2.224 & $d_{0}$-Rydberg \\
\hline 57 & $a_{1}$ & 2.337 & $d_{+2}$-Rydberg \\
\hline 58 & $a_{2}$ & 2.352 & $d_{-2}$-Rydberg \\
\hline 59 & $b_{1}$ & 2.390 & $d_{+1}$-Rydberg \\
\hline 60 & $b_{1}$ & 2.702 & $\pi_{3}^{*}$ \\
\hline 61 & $b_{2}$ & 2.826 & $p_{y}-$ Rydberg \\
\hline 65 & $a_{2}$ & 3.442 & $\pi_{4}^{*}$ \\
\hline 70 & $b_{2}$ & 3.927 & $\sigma^{*}$ \\
\hline 72 & $b_{1}$ & 4.219 & $\sigma^{*}$ \\
\hline 74 & $b_{1}$ & 4.375 & $\pi_{5}^{*}$ \\
\hline 79 & $b_{2}$ & 5.167 & $\sigma^{*}$ \\
\hline 82 & $a_{2}$ & 5.649 & $\pi_{6}^{*}$ \\
\hline 84 & $b_{2}$ & 6.438 & $\sigma^{*}$ \\
\hline 95 & $b_{1}$ & 9.421 & $\sigma^{*}$ \\
\hline
\end{tabular}

present SAC/SAC-CI calculations, the active space consists of 17 occupied orbitals and 285 virtual orbitals, only the $1 \mathrm{~s}$ core molecular orbitals (MOs) being frozen. The detailed conditions of the SAC/SAC-CI calculations are exactly the same as those for cyclopentadiene, ${ }^{38}$ furan, and pyrrole. ${ }^{39}$ 
TABLE II. Calculated singlet and triplet excitation energies $\Delta E$ (in eV), oscillator strengths $f(r)$, second moments and main configurations by SAC/SAC-CI method for thiophene.

\begin{tabular}{|c|c|c|c|c|c|c|c|}
\hline \multirow[b]{2}{*}{ State } & \multirow[b]{2}{*}{ Main configuration } & \multicolumn{2}{|c|}{ SAC/SAC-CI } & \multicolumn{4}{|c|}{ Second moment } \\
\hline & & $\Delta E(\mathrm{eV})$ & $f(r)$ & $\left\langle x^{2}\right\rangle$ & $\left\langle y^{2}\right\rangle$ & $\left\langle z^{2}\right\rangle$ & $\left\langle r^{2}\right\rangle$ \\
\hline \multicolumn{8}{|l|}{ Singlet } \\
\hline${ }^{1}{ }^{1} A_{1}$ & Ground state & 0 & & 31.1506 & 24.2497 & 27.0050 & 82.4052 \\
\hline $2{ }^{1} A_{1}$ & $0.74(21-60)+0.34(21-74)-0.32(22-82)-0.24(22-65)$ & 5.41 & 0.0911 & 31.7389 & 25.7192 & 27.3151 & 84.7732 \\
\hline $1{ }^{1} A_{2}$ & $-0.50(22-45)+0.47(22-54)+0.43(22-36)$ & 5.70 & 0.0000 & 48.0408 & 42.2990 & 41.6378 & 131.9777 \\
\hline $1^{1} B_{2}$ & $-0.84(22-60)-0.34(22-74)+0.23(22-59)$ & 5.72 & 0.1131 & 32.2070 & 23.7881 & 29.7098 & 85.7050 \\
\hline${ }^{1}{ }^{1} B_{1}$ & $\begin{array}{l}0.41(22-51)-0.35(22-42)-0.32(22-61)-0.28(22-70) \\
-0.26(22-84)-0.26(22-79)\end{array}$ & 5.87 & 0.0112 & 34.8407 & 40.6329 & 35.8586 & 111.3322 \\
\hline $2{ }^{1} A_{2}$ & $\begin{array}{l}-0.38(21-70)-0.33(21-84)-0.32(21-79)+0.31(21-51) \\
+0.31(21-55)-0.30(21-61)\end{array}$ & 6.03 & 0.0000 & 31.9277 & 34.1791 & 33.7306 & 99.8375 \\
\hline $2{ }^{1} B_{1}$ & $0.50(21-45)-0.47(21-54)-0.43(21-36)-0.31(21-27)$ & 6.12 & 0.0001 & 48.5316 & 42.0712 & 41.6709 & 132.2737 \\
\hline $3{ }^{1} A_{2}$ & $0.53(22-43)-0.50(22-52)+0.42(22-34)-0.28(22-63)$ & 6.41 & 0.0000 & 47.0079 & 38.1895 & 77.5094 & 162.7068 \\
\hline $2{ }^{1} B_{2}$ & $0.55(22-44)-0.53(22-53)+0.41(22-35)-0.33(22-64)$ & 6.41 & 0.0376 & 80.6863 & 35.5601 & 39.3059 & 155.5523 \\
\hline $3{ }^{1} B_{1}$ & $\begin{array}{l}-0.41(22-42)-0.35(22-33)-0.35(22-55)+0.35(22-70) \\
+0.30(22-46)+0.30(22-51)\end{array}$ & 6.47 & 0.0165 & 43.5744 & 64.8495 & 46.8936 & 155.3175 \\
\hline $3{ }^{1} A_{1}$ & $0.54(21-44)-0.52(21-53)+0.39(21-35)+0.33(21-64)$ & 6.73 & 0.0655 & 77.8235 & 37.2392 & 36.4978 & 151.5604 \\
\hline $4{ }^{1} A_{2}$ & $-0.50(22-57)-0.47(22-49)+0.31(22-40)+0.29(22-69)$ & 6.73 & 0.0000 & 52.5661 & 43.2566 & 49.1488 & 144.9715 \\
\hline $4{ }^{1} B_{1}$ & $-0.55(21-43)+0.51(21-52)-0.42(21-34)+0.28(21-63)$ & 6.73 & 0.0193 & 46.1183 & 42.4244 & 75.9815 & 164.5242 \\
\hline $5{ }^{1} A_{2}$ & $-0.52(22-47)+0.48(22-56)-0.39(22-38)-0.29(22-71)$ & 6.75 & 0.0000 & 41.3948 & 53.8679 & 63.3268 & 158.5895 \\
\hline $6{ }^{1} A_{2}$ & $0.48(21-42)+0.40(21-33)-0.35(21-51)-0.26(21-70)$ & 6.89 & 0.0000 & 46.4728 & 79.0322 & 41.7282 & 167.2332 \\
\hline $4{ }^{1} A_{1}$ & $-0.58(22-48)-0.56(22-39)+0.39(22-58)+0.28(22-30)$ & 7.08 & 0.0177 & 91.7148 & 81.2631 & 42.2013 & 215.1792 \\
\hline $3{ }^{1} B_{2}$ & $0.57(22-41)+0.56(22-50)-0.35(22-59)-0.30(22-31)$ & 7.12 & 0.0033 & 96.1437 & 39.1037 & 89.8120 & 225.0594 \\
\hline $5{ }^{1} B_{1}$ & $-0.46(21-57)-0.46(21-49)+0.33(21-40)+0.28(21-69)$ & 7.14 & 0.0001 & 52.5975 & 36.0913 & 67.5834 & 156.2721 \\
\hline $6{ }^{1} B_{1}$ & $0.51(22-37)+0.45(22-46)-0.28(22-29)+0.24(22-33)$ & 7.15 & 0.0000 & 52.1380 & 94.2201 & 81.2187 & 227.5768 \\
\hline $7{ }^{1} B_{1}$ & $-0.52(21-47)+0.44(21-56)-0.44(21-38)-0.25(21-71)$ & 7.21 & 0.0000 & 46.2803 & 85.0816 & 46.0036 & 177.3655 \\
\hline $7{ }^{1} A_{2}$ & $0.56(22-27)-0.47(22-23)-0.36(22-54)+0.22(22-36)$ & 7.23 & 0.0000 & 151.6530 & 111.8982 & 92.8254 & 356.3766 \\
\hline $5^{1} A_{1}$ & $0.57(22-65)+0.46(22-82)+0.34(21-60)-0.20(21-59)$ & 7.32 & 0.3614 & 48.5737 & 33.9397 & 36.3434 & 118.8568 \\
\hline $4{ }^{1} B_{2}$ & $-0.54(21-65)-0.48(21-82)+0.25(22-26)+0.24(21-48)$ & 7.40 & 0.1204 & 82.7311 & 48.9592 & 45.0122 & 176.7025 \\
\hline $5{ }^{1} B_{2}$ & $0.43(22-26)-0.42(21-48)-0.41(21-39)-0.40(22-35)$ & 7.43 & 0.0039 & 172.4345 & 89.4303 & 68.1378 & 330.0026 \\
\hline $8{ }^{1} B_{1}$ & $0.62(22-24)-0.45(22-33)-0.36(22-51)-0.22(22-29)$ & 7.45 & 0.0047 & 107.1687 & 252.7777 & 112.2372 & 472.1836 \\
\hline $8{ }^{1} A_{2}$ & $0.62(22-25)-0.52(22-34)-0.36(22-52)-0.24(22-63)$ & 7.46 & 0.0000 & 109.1968 & 94.4712 & 252.2310 & 455.8990 \\
\hline $6{ }^{1} B_{2}$ & $\begin{array}{l}0.38(21-65)+0.38(22-26)+0.35(21-39)+0.33(21-82) \\
-0.32(22-35)+0.31(21-48)\end{array}$ & 7.46 & 0.0236 & 140.4010 & 73.7045 & 61.4728 & 275.5784 \\
\hline $6{ }^{1} A_{1}$ & $-0.58(21-41)-0.55(21-50)+0.31(21-59)+0.29(21-31)$ & 7.47 & 0.0336 & 96.5094 & 42.5028 & 87.3996 & 226.4118 \\
\hline $9{ }^{1} A_{2}$ & $-0.48(22-32)+0.45(22-40)+0.29(22-28)-0.26(22-38)$ & 7.54 & 0.0000 & 114.9392 & 75.1133 & 230.1971 & 420.2497 \\
\hline $10{ }^{1} A_{2}$ & $0.60(22-28)-0.43(22-38)+0.28(22-32)-0.27(22-56)$ & 7.59 & 0.0000 & 116.0788 & 231.7181 & 119.9996 & 467.7964 \\
\hline $9{ }^{1} B_{1}$ & $0.55(21-27)-0.45(21-23)-0.36(21-54)+0.23(21-40)$ & 7.61 & 0.0004 & 166.4129 & 107.4242 & 96.6257 & 370.4628 \\
\hline $10^{1} B_{1}$ & $-0.75(22-29)-0.31(22-46)+0.25(22-55)+0.20(22-37)$ & 7.68 & 0.0001 & 109.9132 & 261.1500 & 247.5259 & 618.5892 \\
\hline $7{ }^{1} B_{2}$ & $0.82(22-31)+0.35(22-50)-0.29(22-59)-0.13(22-41)$ & 7.69 & 0.0020 & 293.3505 & 104.5606 & 286.1196 & 684.0307 \\
\hline $7{ }^{1} A_{1}$ & $-0.80(22-30)-0.33(22-48)+0.32(22-58)+0.20(22-39)$ & 7.70 & 0.0006 & 277.8417 & 261.4545 & 104.3382 & 643.6344 \\
\hline $8{ }^{1} A_{1}$ & $0.63(21-26)-0.52(21-35)-0.36(21-53)-0.27(21-64)$ & 7.79 & 0.0002 & 268.5184 & 106.1164 & 98.3973 & 473.0322 \\
\hline $11^{1} B_{1}$ & $-0.64(21-25)+0.48(21-34)+0.36(21-52)+0.23(21-63)$ & 7.80 & 0.0072 & 110.5075 & 109.1804 & 267.9629 & 487.6508 \\
\hline $8{ }^{1} B_{2}$ & $-0.71(22-26)-0.48(22-35)-0.28(22-44)-0.25(22-53)$ & 7.85 & 0.0033 & 611.5824 & 210.3437 & 215.6045 & 1037.5305 \\
\hline $12{ }^{1} B_{1}$ & $0.56(20-60)+0.34(21-32)-0.34(21-40)+0.27(20-74)$ & 7.86 & 0.0004 & 96.5793 & 108.1910 & 140.5064 & 345.2767 \\
\hline $13^{1} B_{1}$ & $0.60(22-24)+0.51(22-33)+0.28(22-51)+0.19(22-42)$ & 7.87 & 0.0035 & 202.0923 & 529.8150 & 224.2007 & 956.1080 \\
\hline $14^{1} B_{1}$ & $-0.49(21-32)-0.45(21-28)+0.33(21-40)+0.25(21-38)$ & 7.95 & 0.0001 & 143.8030 & 266.6761 & 82.4519 & 492.9310 \\
\hline $9{ }^{1} B_{2}$ & $-0.82(21-30)-0.35(21-48)+0.32(21-58)+0.17(21-39)$ & 8.02 & 0.0001 & 288.0977 & 278.1591 & 105.9431 & 672.1999 \\
\hline $15^{1} B_{1}$ & $0.68(22-37)+0.46(22-29)+0.31(22-55)-0.19(22-62)$ & 8.03 & 0.0000 & 143.7143 & 362.6991 & 351.5673 & 857.9807 \\
\hline $9{ }^{1} A_{1}$ & $-0.59(21-31)-0.49(22-39)-0.31(22-30)-0.25(22-58)$ & 8.06 & 0.0008 & 322.3675 & 220.6878 & 207.0026 & 750.0579 \\
\hline $10^{1} B_{2}$ & $-0.74(22-41)-0.40(22-31)-0.38(22-59)+0.23(22-50)$ & 8.07 & 0.0007 & 365.0271 & 129.1955 & 355.0587 & 849.2814 \\
\hline $10{ }^{1} A_{1}$ & $-0.56(21-31)+0.53(22-39)+0.32(22-30)+0.28(22-58)$ & 8.08 & 0.0001 & 336.2777 & 244.0851 & 203.0907 & 783.4536 \\
\hline $11^{1} A_{1}$ & $-0.68(21-26)-0.50(21-35)-0.28(21-44)-0.27(21-53)$ & 8.19 & 0.0004 & 595.9895 & 209.8464 & 207.3958 & 1013.2318 \\
\hline $11^{1} B_{2}$ & $0.73(22-44)-0.47(22-35)+0.32(22-64)+0.20(22-53)$ & 8.20 & 0.0069 & 602.2870 & 207.4578 & 212.6442 & 1022.3890 \\
\hline $16{ }^{1} B_{1}$ & $-0.67(22-42)+0.40(22-33)+0.37(22-62)-0.25(22-51)$ & 8.21 & 0.0030 & 214.9969 & 527.3109 & 221.7682 & 964.0761 \\
\hline $17^{1} B_{1}$ & $0.63(21-25)+0.53(21-34)+0.29(21-52)+0.23(21-43)$ & 8.22 & 0.0020 & 219.8853 & 224.5123 & 585.4795 & 1029.8772 \\
\hline $18^{1} B_{1}$ & $-0.60(21-32)-0.51(21-40)+0.25(21-23)+0.22(21-27)$ & 8.30 & 0.0001 & 296.4145 & 275.6246 & 413.3376 & 985.3767 \\
\hline $19{ }^{1} B_{1}$ & $-0.64(21-38)-0.53(21-28)-0.29(21-56)+0.19(21-71)$ & 8.33 & 0.0006 & 153.0923 & 449.9668 & 292.1696 & 895.2287 \\
\hline $20{ }^{1} B_{1}$ & $-0.54(22-62)+0.42(22-61)-0.34(21-27)+0.23(22-46)$ & 8.38 & 0.0001 & 235.0066 & 217.8257 & 245.5606 & 698.3930 \\
\hline $12^{1} B_{2}$ & $-0.73(21-39)-0.42(21-30)-0.39(21-58)+0.21(21-48)$ & 8.40 & 0.0000 & 371.9684 & 362.9376 & 132.7741 & 867.6801 \\
\hline $12{ }^{1} A_{1}$ & $0.71(21-41)+0.45(21-31)+0.36(21-59)-0.22(21-50)$ & 8.41 & 0.0003 & 379.6400 & 137.3506 & 368.4652 & 885.4558 \\
\hline $13{ }^{1} A_{1}$ & $0.71(21-44)-0.48(21-35)+0.32(21-64)+0.20(21-53)$ & 8.53 & 0.0018 & 606.5278 & 213.3733 & 211.1042 & 1031.0053 \\
\hline $14{ }^{1} A_{1}$ & $-0.64(22-48)-0.53(22-58)+0.25(22-75)+0.21(19-60)$ & 8.66 & 0.0001 & 241.0693 & 223.5991 & 95.5606 & 560.2290 \\
\hline $13^{1} B_{2}$ & $-0.66(22-50)-0.59(22-59)+0.25(22-81)+0.18(22-41)$ & 8.70 & 0.0002 & 251.2184 & 90.4596 & 242.9286 & 584.6066 \\
\hline $15^{1} A_{1}$ & $-0.51(20-45)+0.46(20-54)+0.43(20-36)+0.31(20-27)$ & 8.77 & 0.0426 & 58.4541 & 49.5909 & 43.8834 & 151.9285 \\
\hline
\end{tabular}


TABLE II. (Continued.)

\begin{tabular}{|c|c|c|c|c|c|c|c|}
\hline \multirow[b]{2}{*}{ State } & \multirow[b]{2}{*}{ Main configuration } & \multicolumn{2}{|c|}{ SAC/SAC-CI } & \multicolumn{4}{|c|}{ Second moment } \\
\hline & & $\Delta E(\mathrm{eV})$ & $f(r)$ & $\left\langle x^{2}\right\rangle$ & $\left\langle y^{2}\right\rangle$ & $\left\langle z^{2}\right\rangle$ & $\left\langle r^{2}\right\rangle$ \\
\hline $14{ }^{1} B_{2}$ & $0.35(22-74)+0.33(22-64)-0.31(22-53)+0.28(20-51)$ & 8.94 & 0.1281 & 108.5872 & 61.1085 & 62.1107 & 231.8064 \\
\hline $15^{1} B_{2}$ & $0.57(22-64)-0.54(22-53)-0.20(22-74)-0.20(22-85)$ & 9.02 & 0.0087 & 247.0414 & 97.8575 & 95.6634 & 440.5623 \\
\hline $16^{1} B_{2}$ & $0.66(21-48)+0.58(21-58)-0.27(21-75)-0.18(21-39)$ & 9.04 & 0.0023 & 249.1371 & 238.4051 & 92.2909 & 579.8332 \\
\hline $16{ }^{1} A_{1}$ & $0.62(21-50)+0.61(21-59)-0.21(21-81)+0.17(22-65)$ & 9.07 & 0.0017 & 235.1688 & 88.8972 & 226.3588 & 550.4248 \\
\hline $17^{1} B_{2}$ & $0.76(22-74)-0.30(22-60)-0.22(20-51)+0.20(20-42)$ & 9.16 & 0.0225 & 53.7501 & 45.8525 & 47.4389 & 147.0415 \\
\hline $17^{1} A_{1}$ & $0.37(21-64)+0.37(21-74)-0.34(21-53)+0.33(20-43)$ & 9.30 & 0.0157 & 125.1763 & 60.0477 & 78.5895 & 263.8135 \\
\hline $18{ }^{1} A_{1}$ & $0.46(21-64)-0.42(21-53)-0.34(20-43)+0.33(20-52)$ & 9.32 & 0.0162 & 164.4771 & 72.5581 & 87.8323 & 324.8675 \\
\hline $19^{1} A_{1}$ & $-0.53(21-74)+0.34(22-82)-0.32(22-65)+0.32(21-60)$ & 9.38 & 0.0211 & 105.4377 & 60.6730 & 56.8228 & 222.9334 \\
\hline $18{ }^{1} B_{2}$ & $0.84(22-72)+0.23(22-95)-0.22(22-81)-0.17(21-65)$ & 9.44 & 0.0927 & 78.6144 & 36.1895 & 59.0746 & 173.8785 \\
\hline $20{ }^{1} A_{1}$ & $0.51(22-82)+0.46(21-74)-0.33(22-65)-0.28(19-60)$ & 9.56 & 0.0390 & 74.5562 & 48.8127 & 48.8346 & 172.2035 \\
\hline $19^{1} B_{2}$ & $0.53(21-65)-0.52(21-82)+0.35(20-42)+0.29(20-33)$ & 9.68 & 0.0002 & 52.4212 & 63.3838 & 44.3538 & 160.1588 \\
\hline $21^{1} A_{1}$ & $0.76(21-72)-0.27(21-74)+0.23(21-95)-0.19(21-81)$ & 9.78 & 0.0319 & 75.6652 & 40.0539 & 57.4349 & 173.1540 \\
\hline $22{ }^{1} A_{1}$ & $-0.47(20-47)+0.41(20-56)-0.34(20-38)-0.25(21-72)$ & 9.85 & 0.1378 & 52.7586 & 54.9992 & 74.1498 & 181.9075 \\
\hline \multicolumn{8}{|l|}{ Triplet } \\
\hline $1{ }^{3} B_{2}$ & $0.81(22-60)+0.44(22-74)$ & 3.94 & .. & 31.1381 & 23.0524 & 28.6764 & 82.8668 \\
\hline $1{ }^{3} A_{1}$ & $0.78(21-60)+0.40(21-74)+0.27(22-82)$ & 4.86 & .. & 31.4461 & 25.8997 & 26.5303 & 83.8760 \\
\hline $1{ }^{3} A_{2}$ & $0.51(22-45)-0.48(22-54)-0.44(22-36)$ & 5.75 & $\ldots$ & 49.6142 & 41.7454 & 43.4494 & 134.8091 \\
\hline $1^{3} B_{1}$ & $0.43(22-51)-0.38(22-42)-0.32(22-61)$ & 5.94 & $\cdots$ & 35.9006 & 43.7472 & 36.4166 & 116.0643 \\
\hline
\end{tabular}

\section{RESULTS AND DISCUSSION}

We show some important SCF orbitals in Table I. The four occupied MOs in the highest occupied molecular orbital (HOMO) region are $\pi$ - and lone-pair orbitals. In the unoccupied MOs, many Rydberg MOs appear first and the valence MOs appear in energy higher than $2.7 \mathrm{eV}$. The total SCF energy of thiophene is calculated to be -551.376387 Hartree. The SAC ground-state energy is -551.667755 Hartree, and the correlation energy is -0.291368 Hartree.

The valence and Rydberg transitions are distinguished by the criteria discussed in our previous article ${ }^{38}$ for the $C_{2 V}$ five-membered ring compounds. First, the $A_{2}$ and $B_{1}$ Rydberg states are not perturbed by the valence $\pi-\pi^{*}$ transitions. Second, the second moment of the charge distribution $\left\langle r^{2}\right\rangle$, and its components $\left\langle x^{2}\right\rangle,\left\langle y^{2}\right\rangle$, and $\left\langle z^{2}\right\rangle$ give the size of the electron cloud of the state under consideration. In addition, the oscillator strength has also been used by some authors $^{30-32,34,35}$ as an indicator to distinguish valence with Rydberg states.

The Rydberg transitions originating from the $1 a_{2}$ orbital (HOMO) are substantially different from those originating from the $3 b_{1}$ orbital (next HOMO, see Table I), since the $1 a_{2}$ orbital has a node at the sulfur atom. For convenience, they are labeled as $R$ and $R^{\prime}$, respectively, in the present study.

The calculated excitation energies, oscillator strengths, main configurations, and second moments are shown in Table II. The calculated excitation energies which are used to assign the experimental data are shown in Table III, together with the other theoretical results by the CASPT2 and MRD-CI methods.

\section{A. Energy range lower than $5 \mathrm{eV}$}

The onset of the optical absorption in thiophene is located at $5 \mathrm{eV}$. Two lowest triplet transitions have been identified by the EEL ${ }^{19-21}$ spectra at 3.8 and $4.7 \mathrm{eV}$, which are assigned to the ${ }^{3} B_{2}$ and ${ }^{3} A_{1}$ valence transitions, respectively. Our present calculations computed the $1{ }^{3} B_{2}$ state at $3.94 \mathrm{eV}$ and the $1{ }^{3} A_{1}$ state at $4.86 \mathrm{eV}$. The present SAC-CI triplet excitation energies are in good agreement with experimental values. Calculated second moments of these two states are 82.9 a.u. $^{2}$ and 83.9 a.u. ${ }^{2}$, and these clearly characterize $1^{3} B_{2}$ and $1^{3} A_{1}$ as valence $\pi-\pi^{*}$ excitations. The main configurations of $1^{3} B_{2}$ are $(22 \rightarrow 60)$ and $(22 \rightarrow 74)$, and those of $1^{3} A_{1}$ are $(21 \rightarrow 60),(21 \rightarrow 74)$, and $(22 \rightarrow 82)$. CASPT2 gives the $1{ }^{3} B_{2}$ and $1{ }^{3} A_{1}$ states at 3.75 and $4.50 \mathrm{eV}$, respectively. MRD-CI gives the corresponding states at 4.45 and $5.03 \mathrm{eV}$, respectively. We also calculated two other triplet transitions, $1^{3} A_{2}$ and $1{ }^{3} B_{2}$, at 5.75 and $5.94 \mathrm{eV}$, respectively. The corresponding states of CASPT2 calculations were given at 5.88 and $5.90 \mathrm{eV}$, respectively, which are close to the SAC-CI results.

\section{B. Energy range 5-6.5 eV}

The first VUV band (see Fig. 2, historically denoted as bands $\mathrm{A}$ and $\mathrm{B}$ ) is located in this energy region. The fine structure on the rising side of band A was suggested to originate from the valence state with vibrational structures. Moreover, the MCD spectrum of thiophene shows two bands at 5.27 and $5.64 \mathrm{eV}$, respectively, with opposite signs in their $B$-values, ${ }^{16-18}$ confirming the presence of two valence $\pi-\pi^{*}$ excitations. Our present calculations support the above MCD experimental conclusion: we obtained the $2{ }^{1} A_{1}$ and $1{ }^{1} B_{2}$ states with the excitation energies computed at 5.41 and 5.72 $\mathrm{eV}$ with the oscillator strength 0.0911 and 0.1131 , respectively, that are clearly responsible for the intensity of the first VUV band. The second moments $\left(\left\langle r^{2}\right\rangle\right)$ of the two states are 84.8 and 85.7 a.u. ${ }^{2}$, respectively, which clearly define both of them as valence excited states comparing with that of the ground state $\left(\left\langle r^{2}\right\rangle=82.4\right.$ a.u. $)$. The main configurations of the $2{ }^{1} A_{1}$ state are $\pi-\pi^{*}$ excitations of $21 \rightarrow 60,21 \rightarrow 74$, $22 \rightarrow 82$, and $22 \rightarrow 65$, etc., and those of the $1{ }^{1} B_{2}$ state are 
TABLE III. SAC-CI results compared with the experimental excitation energies (in eV) and other theoretical results for thiophene.

\begin{tabular}{|c|c|c|c|c|c|c|c|c|c|c|}
\hline \multirow[b]{2}{*}{ State } & \multirow[b]{2}{*}{ Nature } & \multicolumn{2}{|c|}{ SAC/SAC-CI } & \multirow[b]{2}{*}{ Expt. $^{a}$} & \multicolumn{2}{|c|}{$\mathrm{CASPT}^{\mathrm{b}}$} & \multicolumn{2}{|c|}{ MRD-CI } & \multicolumn{2}{|c|}{$\mathrm{CI}^{\mathrm{d}}$} \\
\hline & & $f(r)$ & $\Delta E$ & & $\Delta E$ & $f(r)$ & $\Delta E$ & $f(r)$ & $\Delta E$ & $f(r)$ \\
\hline $1{ }^{1} A_{1}$ & Ground state & & 0 & & & & & & & \\
\hline $2{ }^{1} A_{1}$ & Valence & 0.0911 & 5.41 & 5.5 & 5.33 & 0.089 & $5.69^{\mathrm{e}}$ & 0.119 & 6.67 & 0.068 \\
\hline $1{ }^{1} A_{2}$ & $3 s$ Ryd. & forbidden & 5.70 & 6.0 & 5.93 & forbidden & 5.779 & forbidden & & \\
\hline $1{ }^{1} B_{2}$ & Valence & 0.1131 & 5.72 & 5.5 & 5.72 & 0.070 & $6.00^{\mathrm{e}}$ & 0.154 & 7.74 & 0.047 \\
\hline $1{ }^{1} B_{1}$ & $1 a_{2}-\sigma^{*}$ & 0.0112 & 5.87 & 6.0 & & & 6.412 & & & \\
\hline $2{ }^{1} A_{2}$ & $3 b_{1}-\sigma^{*}$ & forbidden & 6.03 & 6.0 & & & & & & \\
\hline $2{ }^{1} B_{1}$ & $3 s^{\prime}$ Ryd. & 0.0001 & 6.12 & 6.0 & 6.23 & 0.0002 & 6.334 & 0.000 & & \\
\hline $3{ }^{1} A_{2}$ & $3 p_{z}$ Ryd. & forbidden & 6.41 & 6.606 & 6.58 & forbidden & 7.028 & forbidden & & \\
\hline $2{ }^{1} B_{2}$ & $3 p_{x}$ Ryd. & 0.0376 & 6.41 & 6.606 & 6.56 & 0.0296 & 7.098 & 0.034 & & \\
\hline $3{ }^{1} B_{1}$ & $3 p_{y}$ Ryd. & 0.0165 & 6.47 & 6.606 & 6.30 & 0.0303 & 6.387 & 0.000 & & \\
\hline $3{ }^{1} A_{1}$ & $3 p_{x}^{\prime}$ Ryd. & 0.0655 & 6.73 & $6.7-7.0$ & 6.76 & 0.0150 & 7.311 & 0.021 & & \\
\hline $4{ }^{1} A_{2}$ & $3 d_{+2}$ Ryd. & forbidden & 6.73 & & 6.97 & forbidden & 7.594 & forbidden & & \\
\hline $4{ }^{1} B_{1}$ & $3 p_{z}^{\prime}$ Ryd. & 0.0193 & 6.73 & $6.7-7.0$ & 6.83 & 0.0200 & 7.175 & 0.029 & & \\
\hline $5{ }^{1} A_{2}$ & $3 d_{0}$ Ryd. & forbidden & 6.75 & & 7.08 & forbidden & 7.853 & forbidden & & \\
\hline $6{ }^{1} A_{2}$ & $3 p_{y}^{\prime}$ Ryd. & forbidden & 6.89 & $6.7-7.0$ & 6.35 & forbidden & & & & \\
\hline $4{ }^{1} A_{1}$ & $3 d_{-2}$ Ryd. & 0.0177 & 7.08 & & 7.23 & 0.0006 & 7.926 & 0.001 & & \\
\hline $3{ }^{1} B_{2}$ & $3 d_{+1}$ Ryd. & 0.0033 & 7.12 & & 7.28 & 0.0005 & 7.760 & 0.000 & & \\
\hline $5{ }^{1} B_{1}$ & $3 d_{+2}^{\prime}$ Ryd. & 0.0001 & 7.14 & & 7.37 & 0.0011 & & & & \\
\hline $6{ }^{1} B_{1}$ & $3 d_{-1}$ Ryd. & 0.0000 & 7.15 & & 7.24 & 0.0010 & & & & \\
\hline $7{ }^{1} B_{1}$ & $3 d_{0}^{\prime}$ Ryd. & 0.0000 & 7.21 & & 7.67 & 0.0001 & & & & \\
\hline $7{ }^{1} A_{2}$ & 4s Ryd. & forbidden & 7.23 & & & & 7.624 & forbidden & & \\
\hline $5{ }^{1} A_{1}$ & Valence & 0.3614 & 7.32 & 7.05 & 6.69 & 0.185 & $7.91^{\mathrm{e}}$ & 0.429 & 9.05 & 0.172 \\
\hline $4{ }^{1} B_{2}$ & Valence & 0.1204 & 7.40 & 7.05 & 7.32 & 0.392 & $8.10^{\mathrm{e}}$ & 0.131 & 9.81 & 0.486 \\
\hline $5{ }^{1} B_{2}$ & $4 p_{x} / 3 d_{-2}^{\prime}$ Ryd. & 0.0039 & 7.43 & 7.786 & & & & & & \\
\hline $8{ }^{1} B_{1}$ & $4 P_{y}$ Ryd. & 0.0047 & 7.45 & 7.786 & & & & & & \\
\hline $8{ }^{1} A_{2}$ & $4 P_{z}$ Ryd. & forbidden & 7.46 & 7.786 & & & & & & \\
\hline $6{ }^{1} B_{2}$ & $3 d_{-2}^{\prime} / 4 p_{x}$ Ryd. & 0.0236 & 7.46 & & 7.53 & 0.0022 & & & & \\
\hline $6{ }^{1} A_{1}$ & $3 d_{+1}^{\prime}$ Ryd & 0.0336 & 7.47 & & 7.57 & 0.0001 & & & & \\
\hline $9{ }^{1} A_{2}$ & $4 d_{+2}$ Ryd. & forbidden & 7.54 & 8.006 & & & & & & \\
\hline $10{ }^{1} A_{2}$ & $4 d_{0}$ Ryd. & forbidden & 7.59 & 8.006 & & & & & & \\
\hline $9{ }^{1} B_{1}$ & $4 s^{\prime}$ Ryd. & 0.0004 & 7.61 & 8.14 & & & & & & \\
\hline $10{ }^{1} B_{1}$ & $4 d_{-1}$ Ryd. & 0.0001 & 7.68 & 8.006 & & & & & & \\
\hline $7{ }^{1} B_{2}$ & $4 d_{+1}$ Ryd. & 0.0020 & 7.69 & 8.006 & & & & & & \\
\hline $7{ }^{1} A_{1}$ & $4 d_{-2}$ Ryd. & 0.0006 & 7.70 & 8.006 & & & & & & \\
\hline $8{ }^{1} A_{1}$ & $4 p_{x}^{\prime}$ Ryd. & 0.0002 & 7.79 & 8.384 & & & & & & \\
\hline $11^{1} B_{1}$ & $4 p_{z}^{\prime}$ Ryd. & 0.0072 & 7.80 & 8.384 & & & & & & \\
\hline $8{ }^{1} B_{2}$ & $5 p_{x}$ Ryd. & 0.0033 & 7.85 & 8.221 & & & & & & \\
\hline $12{ }^{1} B_{1}$ & $n-\pi^{*} / 4 d_{+2}^{\prime}$ Ryd. & 0.0004 & 7.86 & & 7.77 & 0.0333 & & & & \\
\hline $13^{1} B_{1}$ & $5 p_{y}$ Ryd. & 0.0035 & 7.87 & 8.221 & & & & & & \\
\hline $14{ }^{1} B_{1}$ & $4 d_{+2}^{\prime}$ Ryd. & 0.0001 & 7.95 & 7.949 & & & & & & \\
\hline $9{ }^{1} B_{2}$ & $4 d_{-2}^{\prime}$ Ryd. & 0.0001 & 8.02 & 7.949 & & & & & & \\
\hline $15^{1} B_{1}$ & $5 d_{-1}$ Ryd. & 0.0000 & 8.03 & 8.317 & & & & & & \\
\hline $9{ }^{1} A_{1}$ & $4 d_{+1}^{\prime} / 5 d_{-2}$ Ryd. & 0.0008 & 8.06 & & & & & & & \\
\hline $10{ }^{1} B_{2}$ & $5 d_{+1}$ Ryd. & 0.0007 & 8.07 & 8.317 & & & & & & \\
\hline $10{ }^{1} A_{1}$ & $4 d_{+1}^{\prime} / 5 d_{-2}$ Ryd. & 0.0001 & 8.08 & & & & & & & \\
\hline $11{ }^{1} A_{1}$ & $5 p_{x}^{\prime}$ Ryd. & 0.0004 & 8.19 & & & & & & & \\
\hline $11{ }^{1} B_{2}$ & $6 p_{x}$ Ryd. & 0.0069 & 8.20 & 8.436 & & & & & & \\
\hline $16{ }^{1} B_{1}$ & $6 p_{y}$ Ryd. & 0.0030 & 8.21 & 8.436 & & & & & & \\
\hline $17^{1} B_{1}$ & $5 p_{z}^{\prime}$ Ryd. & 0.0020 & 8.22 & & & & & & & \\
\hline $18^{1} B_{1}$ & $5 d_{+2}^{\prime} / 5 s^{\prime}$ Ryd. & 0.0001 & 8.30 & & & & & & & \\
\hline $19^{1} B_{1}$ & $4 d_{0}^{\prime}$ Ryd. & 0.0006 & 8.33 & & & & & & & \\
\hline $20{ }^{1} B_{1}$ & $7 p_{y} / 5 s^{\prime}$ Ryd. & 0.0001 & 8.38 & & & & & & & \\
\hline $12{ }^{1} B_{2}$ & $5 d_{-2}^{\prime}$ Ryd. & 0.0000 & 8.40 & & & & & & & \\
\hline $12{ }^{1} A_{1}$ & $5 d_{+1}^{\prime}$ Ryd. & 0.0003 & 8.41 & 8.559 & & & & & & \\
\hline $13{ }^{1} A_{1}$ & $6 p_{x}^{\prime}$ Ryd. & 0.0018 & 8.53 & & & & & & & \\
\hline $14{ }^{1} A_{1}$ & $6 d_{-2}$ Ryd. & 0.0001 & 8.66 & 8.486 & & & & & & \\
\hline $13{ }^{1} B_{2}$ & $6 d_{+1}$ Ryd. & 0.0002 & 8.70 & 8.486 & & & & & & \\
\hline $15^{1} A_{1}$ & $n-s$ Ryd. & 0.0426 & 8.77 & & 9.10 & 0.0479 & & & & \\
\hline $14{ }^{1} B_{2}$ & V. $17 p_{x}$ Ryd. & 0.1281 & 8.94 & & & & & & & \\
\hline $15^{1} B_{2}$ & $7 p_{x}$ Ryd./V. & 0.0087 & 9.02 & & & & & & & \\
\hline $16{ }^{1} B_{2}$ & $6 d_{-2}^{\prime}$ Ryd. & 0.0023 & 9.04 & & & & & & & \\
\hline $16{ }^{1} A_{1}$ & $6 d_{+1}^{\prime}$ Ryd. & 0.0017 & 9.07 & & & & & & & \\
\hline
\end{tabular}


TABLE III. (Continued.)

\begin{tabular}{|c|c|c|c|c|c|c|c|c|c|c|}
\hline \multirow[b]{2}{*}{ State } & \multirow[b]{2}{*}{ Nature } & \multicolumn{2}{|c|}{ SAC/SAC-CI } & \multirow[b]{2}{*}{ Expt. $^{a}$} & \multicolumn{2}{|c|}{$\mathrm{CASPT}^{\mathrm{b}}$} & \multicolumn{2}{|c|}{$\mathrm{MRD}-\mathrm{CI}^{\mathrm{c}}$} & \multicolumn{2}{|c|}{$\mathrm{CI}^{\mathrm{d}}$} \\
\hline & & $f(r)$ & $\Delta E$ & & $\Delta E$ & $f(r)$ & $\Delta E$ & $f(r)$ & $\Delta E$ & $f(r)$ \\
\hline $17{ }^{1} B_{2}$ & V./7p $p_{y}$ Ryd. & 0.0225 & 9.16 & & & & & & & \\
\hline $17{ }^{1} A_{1}$ & V. $/ 7 p_{x}^{\prime}$ Ryd. & 0.0157 & 9.30 & & & & & & & \\
\hline $18{ }^{1} A_{1}$ & $7 p_{x}^{\prime} / n-p_{z}$ Ryd. & 0.0162 & 9.32 & & & & & & & \\
\hline $19{ }^{1} A_{1}$ & $3 b_{1}-\pi^{*} / 1 a_{2}-\pi^{*}$ & 0.0211 & 9.38 & & & & & & & \\
\hline $18{ }^{1} B_{2}$ & $\pi-\sigma^{*}$ & 0.0927 & 9.44 & & & & & & & \\
\hline $20{ }^{1} A_{1}$ & $1 a_{2}-\pi^{*} / 3 b_{1}-\pi^{*}$ & 0.0390 & 9.56 & & & & & & & \\
\hline $19^{1} B_{2}$ & $3 b_{1}-\pi^{*} / n-P_{y}$ & 0.0002 & 9.68 & & & & & & & \\
\hline $21{ }^{1} A_{1}$ & $3 b_{1}-\sigma^{*}$ & 0.0319 & 9.78 & & & & & & & \\
\hline $22{ }^{1} A_{1}$ & $n-d_{0}$ Ryd. & 0.1378 & 9.85 & & & & & & & \\
\hline $1{ }^{3} B_{2}$ & Valence & $\cdots$ & 3.94 & 3.8 & 3.75 & $\ldots$ & 4.45 & & & \\
\hline $1{ }^{3} A_{1}$ & Valence & $\cdots$ & 4.86 & 4.7 & 4.50 & $\cdots$ & 5.03 & & & \\
\hline $1{ }^{3} A_{2}$ & $3 s$ Ryd. & $\cdots$ & 5.75 & & 5.88 & $\cdots$ & & $\cdots$ & & \\
\hline $1{ }^{3} B_{1}$ & $3 p_{y} \mathrm{Ryd}$ & $\cdots$ & 5.94 & & 5.90 & $\cdots$ & & $\cdots$ & & \\
\hline
\end{tabular}

${ }^{a}$ References 21-25, 32.

${ }^{\mathrm{b}}$ Reference 29.

${ }^{\mathrm{c}}$ Reference 32.

${ }^{\mathrm{d}}$ Reference 12.

${ }^{\mathrm{e}}$ Results calculated by TZVP basis set, see Ref. 32 .

$\pi-\pi^{*}$ excitations of $22 \rightarrow 60,22 \rightarrow 74$, etc. Our present calculations suggest that band $\mathrm{A}$ is due to these two valence $\pi-\pi^{*}$ transitions and their vibrational structures. CASPT2 calculated the two states at 5.33 and $5.72 \mathrm{eV}$, respectively,

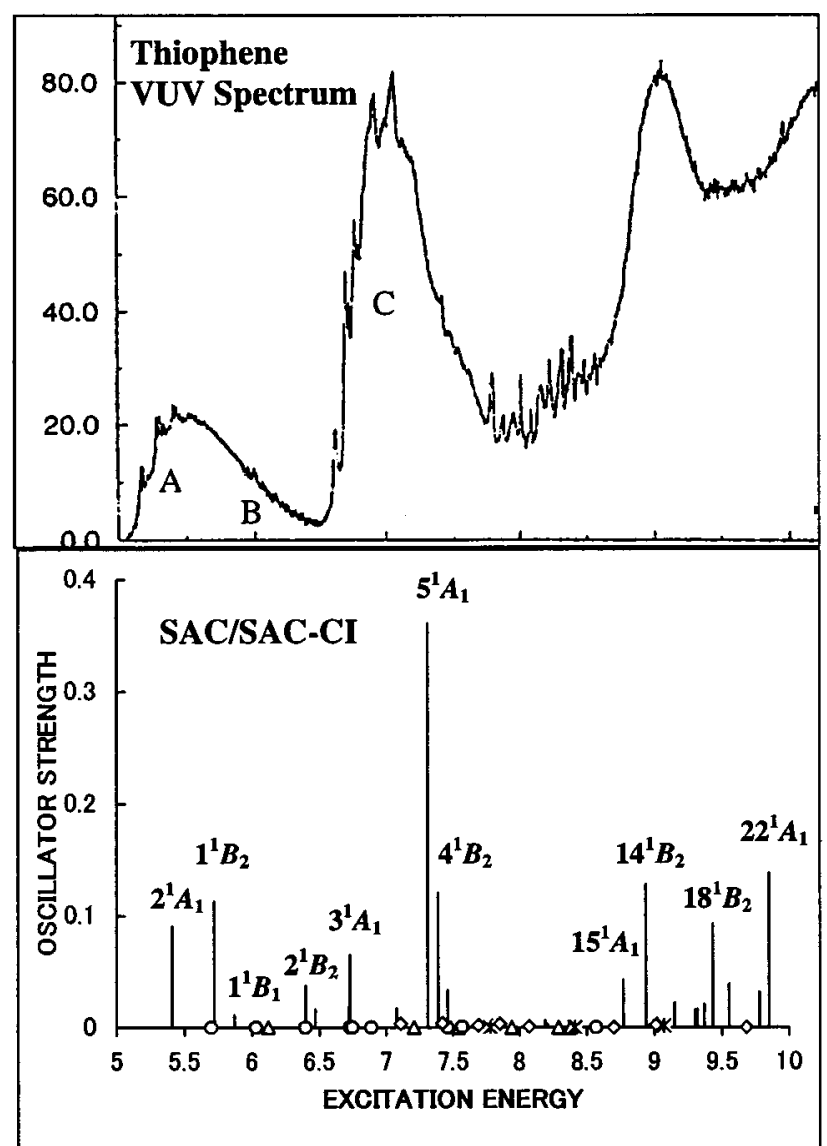

FIG. 2. VUV spectrum and SAC/SAC-CI theoretical spectrum of thiophene. " "*, denotes singlet-excited state with $A_{1}$ symmetry, " $\bigcirc$ " denotes singletexcited state with $A_{2}$ symmetry, " $\triangle$ " denotes singlet-excited state with $B_{1}$ symmetry, and " $\diamond$ " denotes singlet-excited state with $B_{2}$ symmetry. and MRD-CI at 5.69 and $6.00 \mathrm{eV}$, respectively. Note that SAC-CI, CASPT2, and MRD-CI gave very similar theoretical results in this energy region.

The weak fine structure around $6.0 \mathrm{eV}$ (historically called band B) is absent in the condensed-phase spectra, ${ }^{12-14}$ suggesting that this fine structure is Rydberg in nature. Palmer et al. ${ }^{32}$ used the Rydberg equation to assign it to the $1 a_{2} \rightarrow 3 s$ Rydberg transition, though it is symmetryforbidden. In our present calculations, the $1{ }^{1} B_{1}$ state $\left(1 a_{2}\right.$ $\rightarrow \sigma^{*}$ mixed with $1 a_{2} \rightarrow 3 p_{y}$ Rydberg) was calculated at $5.87 \mathrm{eV}$, the $2{ }^{1} A_{2}$ state $\left(3 b_{1} \rightarrow \sigma^{*}\right.$ mixed with $3 b_{1} \rightarrow 4 d_{-1}$ Rydberg) at $6.03 \mathrm{eV}$, suggesting that valence $\pi \rightarrow \sigma^{*}$ transition is a main component of band $\mathrm{B}$. In addition, the $3 s$ and $3 s^{\prime}$ Rydberg transitions were also given in this energy region. The symmetry-forbidden $1{ }^{1} A_{2}$ state $\left(1 a_{2} \rightarrow 3 s\right)$ was given at $5.70 \mathrm{eV}$. The $2{ }^{1} B_{1}$ state $\left(3 b_{1} \rightarrow 3 s\right)$ was given at $6.12 \mathrm{eV}$, suggesting that this weak Rydberg transition might be included in the absorption of band $\mathrm{B}$. Note that $1 a_{2}$ $\rightarrow \sigma^{*}$ and $1 a_{2} \rightarrow \sigma^{*}$-type transitions are not mentioned in the CASPT2 study. MRD-CI calculated the corresponding states at $5.779 \mathrm{eV}\left(1 a_{2} \rightarrow 3 s\right), 6.412 \mathrm{eV}\left(\pi Y+\sigma^{*}\right), 6.852$ $\mathrm{eV}\left(\pi Y Z+\sigma^{*}\right)$, and $6.334 \mathrm{eV}\left(3 b_{1} \rightarrow 3 s\right)$, respectively.

\section{Energy range 6.5-7.7 eV}

The second VUV band (historically called band C) is located in this region, and the maximum of the band is around $7.05 \mathrm{eV}$. The fine structure on the rising side of $\mathrm{C}$ band (around $6.6 \mathrm{eV}$ ) was suggested to associate with $3 p$ Rydberg transitions. ${ }^{5,32}$ Most of the intensity in this band was assumed to originate from the excitations of the further valence $\pi-\pi^{*}$ character. ${ }^{5,32}$ Our present calculations strongly support this assignment.

The $3{ }^{1} A_{2}\left(1 a_{2} \rightarrow 3 p_{z}\right.$ Rydberg $), 2{ }^{1} B_{2}\left(1 a_{2} \rightarrow 3 p_{x}\right.$ Rydberg), and $3{ }^{1} B_{1}\left(1 a_{2} \rightarrow 3 p_{y}\right.$ Rydberg $)$ states were calculated at $6.41,6.41$, and $6.47 \mathrm{eV}$, respectively. We assign this $3 p$ series of Rydberg states to the peak of $6.606 \mathrm{eV}$ since our 
TABLE IV. Excitation energies (in eV) and oscillator strengths calculated by the SAC-CI method for the valence excitations of cyclopentadiene (CP), furan, pyrrole, and thiophene.

\begin{tabular}{|c|c|c|c|c|c|c|c|c|}
\hline \multirow[b]{2}{*}{ State } & \multicolumn{2}{|c|}{$\mathrm{CP}$} & \multicolumn{2}{|c|}{ Furan } & \multicolumn{2}{|c|}{ Pyrrole } & \multicolumn{2}{|c|}{ Thiophene } \\
\hline & $\Delta E$ & $f(r)$ & $\Delta E$ & $f(r)$ & $\Delta E$ & $f(r)$ & $\Delta E$ & $f(r)$ \\
\hline $1{ }^{1} B_{2}$ & 5.54 & 0.1305 & 6.40 & 0.1852 & $6.48^{\mathrm{a}}$ & 0.0475 & 5.72 & 0.1131 \\
\hline $2{ }^{1} A_{1}$ & 6.76 & 0.0009 & 6.79 & 0.0000 & 6.41 & 0.0002 & 5.41 & 0.0911 \\
\hline${ }^{1} A_{1}$ & 8.17 & 0.5377 & 8.34 & 0.4826 & 7.88 & 0.3252 & 7.32 & 0.3614 \\
\hline${ }^{1} B_{2}$ & $\cdots$ & $\ldots$ & 9.08 & 0.1662 & 8.25 & 0.2553 & 7.40 & 0.1204 \\
\hline${ }^{3} B_{2}$ & 3.40 & 0.0 & 4.39 & 0.0 & 4.58 & 0.0 & 3.94 & 0.0 \\
\hline${ }^{3} A_{1}$ & 5.18 & 0.0 & 5.63 & 0.0 & 5.08 & 0.0 & 4.86 & 0.0 \\
\hline
\end{tabular}

${ }^{\text {a This state }}\left(2{ }^{1} B_{2}\right)$ was strongly mixed with Rydberg $3 p$ transitions, see Ref. 39

calculated excitation energies of several lowest Rydberg states are systematically lower than the experimental data, which also occurred in our previous studies for cyclopentadiene, furan, and pyrrole. The $3 p^{\prime}$ Rydberg series were given at $6.73 \mathrm{eV}\left(3{ }^{1} A_{1}, 3 b_{1} \rightarrow 3 p_{x}\right), 6.73 \mathrm{eV}\left(4{ }^{1} B_{1}, 3 b_{1}\right.$ $\left.\rightarrow 3 p_{z}\right)$, and $6.89 \mathrm{eV}\left(6^{1} A_{2}, 3 b_{1} \rightarrow 3 p_{y}\right)$. From our present calculations these $3 p^{\prime}$ Rydberg transitions might also be responsible for the fine structure of the rising side of $\mathrm{C}$ band (up to $7.05 \mathrm{eV}$ ). Derrick et al. ${ }^{5}$ supposed that the first four peaks of $\mathrm{C}$ band are due to $p$-type Rydberg excitations. In agreement with this assignment, Palmer et al. ${ }^{32}$ assigned the peak at $6.606 \mathrm{eV}$ to the $1 a_{2}-3 p$ Rydberg transitions. Our present calculations support these general experimental assignments.

Our present calculations gave the $5{ }^{1} A_{1}$ state at $7.32 \mathrm{eV}$ with oscillator strength 0.3614 , and the $4{ }^{1} B_{2}$ state at 7.40 $\mathrm{eV}$ with oscillator strength 0.1204 , which are the main components of band $\mathrm{C}$. The nature of the $5{ }^{1} A_{1}$ state is a mixture of the $\pi \rightarrow \pi^{*}$ transitions $(22 \rightarrow 65)$ and $(22 \rightarrow 82)$ with $(21 \rightarrow 60)$. The second moment is 118.9 a.u. $^{2}$, a little lager than that of the ground state $\left(82.4\right.$ a.u. $\left.{ }^{2}\right)$. The nature of $4{ }^{1} B_{2}$ state is of strong mixture of $\pi \rightarrow \pi^{*}$ transitions $(21 \rightarrow 65)$ and $(21 \rightarrow 82)$ with Rydberg transitions $(22 \rightarrow 26)$ and $(21 \rightarrow 48)$, and this mixture is responsible for the large second moment $\left(176.7\right.$ a.u. $\left.^{2}\right)$ of this state. We conclude that a significant portion of the electronic absorption intensity of $\mathrm{C}$ band is due to these two valence $\pi \rightarrow \pi^{*}$ excitations $\left(5^{1} A_{1}\right.$ and $4{ }^{1} B_{2}$ ) above-mentioned, especially due to the $5{ }^{1} A_{1}$ valence $\pi \rightarrow \pi^{*}$ transition. Table IV shows clearly that this assignment agrees with the general trend of the most intense absorption band of the other five-membered ring compounds. ${ }^{38,39}$ MRD-CI gave the similar trend of oscillator strengths for these two valence $\pi \rightarrow \pi^{*}$ transitions, though the excitation energies in the MRD-CI study were overestimated.

Note that CASPT2 gave the corresponding valence $\pi \rightarrow \pi^{*}$ excitations of the $3{ }^{1} A_{1}$ state at $6.69 \mathrm{eV}$ with the oscillator strength of 0.1850 obtained by CASSCF state interaction (CASSI) method, and the $4{ }^{1} B_{2}$ state at $7.32 \mathrm{eV}$ with CASSI oscillator strength of 0.3923 . The relative intensities of these two states are calculated oppositely by CASSI to those by SAC-CI and MRD-CI methods. It deserves to be pointed out that a further polarization experimental study, e.g., magnetic circle dichroism study, on the band $\mathrm{C}$ is crucial in the future to verify the relative intensities of the $5{ }^{1} A_{1}$ and $4{ }^{1} B_{2}$ states assigned to $C$ band.
On the higher energy shoulder of $\mathrm{C}$ band, our present calculations gave $3 d$ and $3 d^{\prime}$ Rydberg transitions (see Table III for detailed numerical results). There are no experimental assignments in this energy region, but we propose the existence of the Rydberg states in this energy region of the spectrum.

\section{Energy range $7.7-10 \mathrm{eV}$}

The fine structure in $7.7 \sim 8.8 \mathrm{eV}$ is suggested by the present calculations to represent the Rydberg excitations. Most of them are identified to the two Rydberg series converging to $\mathrm{IP}_{1}\left(1 a_{2}, 8.872 \mathrm{eV}\right)$ and $\mathrm{IP}_{2}\left(3 b_{1}, 9.52 \mathrm{eV}\right)$, respectively. In order to calculate Rydberg states in this region as accurate as possible we use the molecule-centered diffuse functions $(5 s 5 p 5 d)$ in the present calculations. The $4 p, 4 d, 4 s^{\prime}, 4 p^{\prime}, 5 p, 4 d^{\prime}, 6 p, 5 d^{\prime}$, and $6 d$ Rydberg series are computed in this energy region, and the excitation energies and the natures of electronic states are shown in Table III. Since it is the first time to make a theoretical assignment in this far ultraviolet region, there may be room left for some variation in future theoretical and experimental studies.

Palmer et $_{\mathrm{al}}{ }^{32}$ experimentally assigned the absorption at $7.786 \mathrm{eV}$ to $4 p$ Rydberg series, absorption at $7.949 \mathrm{eV}$ to $3 d^{\prime}$ Rydberg, absorption at $8.006 \mathrm{eV}$ to $4 d$ Rydberg series, absorptions at $8.14-8.16 \mathrm{eV}$ to $4 s^{\prime}$ Rydberg series, absorption at $8.221 \mathrm{eV}$ to $5 p$ Rydberg series, absorption at 8.317 $\mathrm{eV}$ to $5 d$ Rydberg series, absorption at $8.384 \mathrm{eV}$ to $4 p^{\prime}$ Rydberg series, absorption at $8.436 \mathrm{eV}$ to $6 p$ Rydberg series, and absorption at $8.486 \mathrm{eV}$ to $6 d$ Rydberg series. Note that this experimental assignment itself was unable to specify, for example, $p_{x}, p_{y}$, and $p_{z}$ Rydberg states. Our present calculations confirm that most of the Rydberg states abovementioned are located at this energy region, and support most of the experimental assignments except for the $3 d^{\prime}$ Rydberg assignment. Our results, as discussed in $6.5-7.7 \mathrm{eV}$ energy region, gave $3 d^{\prime}$ Rydberg series at 7.14-7.47 eV. It is reasonable to assign the absorption $7.949 \mathrm{eV}$ to the $4 d^{\prime}$ Rydberg series, which were computed at $7.86-8.08 \mathrm{eV}$ energy region. We made a comparison between our theoretical assignments and experimental assignments in Table III. We expect that the present results will stimulate new experimental and theoretical studies on the electronic states in this far ultraviolet region.

The excitation of the lone pair electron on the sulfur 
TABLE V. Molecular orbital energies (in eV) and the main configurations of the lowest ${ }^{1} A_{1}$ valence $\pi-\pi^{*}$ excited states of cyclopentadiene, furan, pyrrole, and thiophene.

\begin{tabular}{|c|c|c|c|c|c|}
\hline & & Cyclopentadiene & Furan & Pyrrole & Thiophene \\
\hline $\begin{array}{l}\text { Main } \\
\text { Configuration }\end{array}$ & & $\begin{array}{r}0.54\left(\pi_{1} \rightarrow \pi_{3}^{*}\right) \\
+0.49\left(\pi_{2} \rightarrow \pi_{4}^{*}\right) \\
-0.39\left(\pi_{2} \rightarrow \pi_{6}^{*}\right) \\
-0.15\left(\pi_{1} \rightarrow \pi_{5}^{*}\right)\end{array}$ & $\begin{array}{r}0.58\left(\pi_{1} \rightarrow \pi_{3}^{*}\right) \\
+0.47\left(\pi_{2} \rightarrow \pi_{4}^{*}\right) \\
-0.43\left(\pi_{2} \rightarrow \pi_{6}^{*}\right) \\
+0.24\left(\pi_{1} \rightarrow \pi_{5}^{*}\right)\end{array}$ & $\begin{array}{l}-0.58\left(\pi_{1} \rightarrow \pi_{3}^{*}\right) \\
+0.47\left(\pi_{2} \rightarrow \pi_{4}^{*}\right) \\
-0.40\left(\pi_{2} \rightarrow \pi_{6}^{*}\right)^{\mathrm{a}} \\
-0.26\left(\pi_{1} \rightarrow \pi_{5}^{*}\right)\end{array}$ & $\begin{array}{r}0.74\left(\pi_{1} \rightarrow \pi_{3}^{*}\right) \\
-0.24\left(\pi_{2} \rightarrow \pi_{4}^{*}\right) \\
-0.32\left(\pi_{2} \rightarrow \pi_{6}^{*}\right) \\
+0.34\left(\pi_{1} \rightarrow \pi_{5}^{*}\right)\end{array}$ \\
\hline $\begin{array}{l}\text { Orbital } \\
\text { Energy }(\mathrm{eV})\end{array}$ & $\begin{array}{l}\pi_{1} \\
\pi_{2} \\
\pi_{3}^{*} \\
\pi_{4}^{*} \\
\pi_{5}^{*} \\
\pi_{6}^{*}\end{array}$ & $\begin{aligned}-11.244 & (17 \mathrm{th}) \\
-8.368 & (18 \mathrm{th}) \\
2.938 & (48 \mathrm{th}) \\
4.095 & (54 \mathrm{th}) \\
5.014 & (60 \mathrm{th}) \\
7.362 & (67 \mathrm{th})\end{aligned}$ & $\begin{array}{rr}-10.835 & (17 \mathrm{th}) \\
-8.702 & (18 \mathrm{th}) \\
3.439 & (48 \mathrm{th}) \\
4.043 & (53 \mathrm{rd}) \\
6.298 & (61 \mathrm{st}) \\
6.988 & (64 \mathrm{th})\end{array}$ & $\begin{array}{rc}-9.454 & (17 \text { th }) \\
-8.054 & (18 \text { th }) \\
3.787 & (52 \text { nd }) \\
4.145 & (53 \mathrm{rd}) \\
6.465 & (62 \mathrm{nd}) \\
7.254 & \text { (66th) }\end{array}$ & $\begin{array}{rr}-9.413 & (21 \mathrm{st}) \\
-8.916 & (22 \mathrm{nd}) \\
2.702 & (60 \mathrm{th}) \\
3.442 & (65 \mathrm{th}) \\
4.375 & (74 \mathrm{th}) \\
5.649 & (82 \mathrm{nd})\end{array}$ \\
\hline $\begin{array}{l}\text { Difference } \\
\text { of }(\mathrm{eV})\end{array}$ & $\begin{array}{l}\pi_{3}^{*}-\pi_{1} \\
\pi_{4}^{*}-\pi_{2} \\
\pi_{6}^{*}-\pi_{2} \\
\pi_{5}^{*}-\pi_{1}\end{array}$ & $\begin{array}{l}14.182 \\
12.463 \\
15.730 \\
16.258\end{array}$ & $\begin{array}{l}14.274 \\
12.745 \\
15.690 \\
17.133\end{array}$ & $\begin{array}{l}13.241 \\
12.199 \\
15.308 \\
15.919\end{array}$ & $\begin{array}{l}12.115 \\
12.358 \\
14.565 \\
13.788\end{array}$ \\
\hline
\end{tabular}

${ }^{a}$ The sign of coefficients of the SCF 66th molecular orbital in pyrrole is opposite to those of the corresponding orbitals in other molecules.

atom to the $\pi^{*}$ orbitals have high energy, as expected, but no experimental evidence is available: it has been suggested that the corresponding peak is covered by the $\pi-\pi^{*}$ bands. The lowest $n-\pi^{*}$ state, $12{ }^{1} B_{1}$, is predicted at $7.86 \mathrm{eV}$ in the present calculations. CASPT2 also calculated the corresponding state at $7.77 \mathrm{eV}$.

In the higher energy region of $8.8-10 \mathrm{eV}$, it seems difficult to give a confirmed interpretation since $\mathrm{IP}_{1}$ and $\mathrm{IP}_{2}$ are also located in this region. Our present calculations give some higher valence excitations, $\pi-\pi^{*}$ and $\pi-\sigma^{*}$, etc. in this region, which are more or less influenced by the higher Rydberg states. The detailed results are shown in Tables II and III.

\section{E. Comparison with other five-membered ring compounds}

In Table IV, we make a comparison of the valence singlet and triplet excited states of thiophene with those of cyclopentadiene, furan, and pyrrole for which similar studies have been carried out. Table IV shows that the $2{ }^{1} A_{1}$ state is the lowest singlet excited state in thiophene and its oscillator strength is relatively strong only in thiophene. Therefore, only in thiophene, the $2{ }^{1} A_{1}$ state has been clearly identified by experiments. A good agreement of the theoretical excitation energy and oscillator strength with the experimental ones in the case of thiophene gives an indirect support to our previous assignment of the $2{ }^{1} A_{1}$ state in the other fivemembered ring compounds. ${ }^{38,39}$

Here we qualitatively interpret the different trend of the $2{ }^{1} A_{1}$ states between thiophene and other five-membered ring compounds. To simplify the analysis we assume that the excitation energy of the $2{ }^{1} A_{1}$ state is approximately related to the orbital energy differences in the main configurations. The main configurations of the $2{ }^{1} A_{1}$ states of cyclopentadiene, furan, pyrrole, and thiophene are listed in Table V, together with the orbital energies of the relevant MOs and their differences. In thiophene, $\pi_{1}$ is relatively unstable due to the antibonding nature between $\pi_{1}$ and $3 p_{z}$ of sulfur, while $\pi_{3}^{*}$ becomes stable due to the $3 p_{z}$ bonding nature. Therefore, the orbital energy difference $\Delta E\left(\pi_{3}^{*}-\pi_{1}\right)$ becomes smaller and the $2{ }^{1} A_{1}$ transition is dominated by $\pi_{1} \rightarrow \pi_{3}^{*}(0.74)$ only in thiophene. For this reason, in thiophene, the excitation energy of the $2{ }^{1} A_{1}$ state becomes lowest, comparing with the same states of cyclopentadiene, furan, and pyrrole.

Furthermore, from Table $\mathrm{V}$ we see that the main configurations of the $2{ }^{1} A_{1}$ states of cyclopentadiene, furan, and pyrrole are very similar, and therefore, it is easy to understand that the oscillator strengths of the $2{ }^{1} A_{1}$ states in these three molecules are similar in magnitude. In thiophene, however, the $2{ }^{1} A_{1}$ state is dominated by different main configurations and the weight of configuration $\pi_{1} \rightarrow \pi_{3}^{*}(0.74)$ is much larger than those in the other three molecules $(0.54-$ $0.58)$. This obvious difference gives rise to a larger contribution of the configuration $\left(\pi_{1} \rightarrow \pi_{3}^{*}\right)$ to the transition dipole moment of the $2{ }^{1} A_{1}$ state in thiophene.

Note that the CASPT2 (Ref. 29) study showed that the $2{ }^{1} A_{1}$ state of thiophene has the smallest weight of the doubly excited configurations among these molecules of interest. In the cases of cyclopentadiene, furan, and pyrrole, the contributions of the doubly excited configurations to the $2{ }^{1} A_{1}$ state were large. In SAC-CI calculations, however, the contributions of doubly excited configurations to the $2{ }^{1} A_{1}$ states are small in all four molecules. Nevertheless, both SAC-CI and CASPT2 methods give similar results on the $2{ }^{1} A_{1}$ state of thiophene.

\section{CONCLUSIONS}

An extended investigation of the electronic excitation spectra of thiophene has been carried out by the SAC/ SAC-CI method. To give a satisfactory theoretical interpretation of the VUV and EEL spectra of thiophene, the 70 singlet and four lowest triplet electronic states were computed. The calculated triplet valence $\pi \rightarrow \pi^{*}$ excited states $1^{3} B_{2}(3.94 \mathrm{eV})$ and $1{ }^{3} A_{1}(4.86 \mathrm{eV})$ agree well with the EEL spectroscopic data. Two pairs of singlet valence $\pi \rightarrow \pi^{*}$ excitations were given, which span the general profile of the VUV spectrum lower than $7.7 \mathrm{eV}$. The $2{ }^{1} A_{1}(5.41 \mathrm{eV})$ and 
${ }^{1} B_{2}(5.72 \mathrm{eV})$ states were assigned to cause most of the intensity of the first band of the VUV spectrum. The $5{ }^{1} A_{1}$ $(7.32 \mathrm{eV})$ and $4{ }^{1} B_{2}(7.40 \mathrm{eV})$ states were assigned to constitute most of the second band of the VUV spectrum. Our present results suggest that the intensity of the second band, i.e., $\mathrm{C}$ band, is largely due to the $5{ }^{1} A_{1}$ valence $\pi \rightarrow \pi^{*}$ excited state (see Fig. 2), instead of due to the $4{ }^{1} B_{2}$ valence $\pi \rightarrow \pi^{*}$ excited state suggested by the CASPT2 study. Polarization experiment on the $\mathrm{C}$ band is, therefore, very interesting. In addition, a number of Rydberg states in the energy region of 6.0, 6.6, and 7.7-8.8 eV was assigned according to our present calculations. This study gives the first theoretical assignment in the far ultraviolet region $(7.7-8.8 \mathrm{eV})$ of the spectrum and is expected to provide a theoretical basis for further experimental and theoretical studies on the excited states of thiophene in this energy region.

\section{ACKNOWLEDGMENTS}

We are grateful to Dr. J. Meller and Mr. K. Toyota for their helpful discussions on this subject. This research was supported by a Grant-in-Aid for Scientific Research from the Ministry of Education, Science, Culture, and Sports.

${ }^{1}$ W. C. Price and A. D. Walsh, Proc. R. Soc. London, Ser. A 179, 201 (1941).

${ }^{2}$ L. W. Pickett, N. J. Hoeflich, and T.-C. Liu, J. Am. Chem. Soc. 73, 4865 (1951).

${ }^{3}$ P. A. Mullen and M. K. Orloff, J. Chem. Phys. 51, 2276 (1969).

${ }^{4}$ J. H. D. Eland, Int. J. Mass Spectrom. Ion Phys. 2, 471 (1969).

${ }^{5}$ P. J. Derrick, L. Asbrink, O. Edqvist, B.-O. Jonsson, and E. Lindholm, Int. J. Mass Spectrom. Ion Phys. 6, 161 (1971).

${ }^{6}$ P. J. Derrick, L. Asbrink, O. Edqvist, and E. Lindholm, Spectrochim. Acta, A 27, 2525 (1971).

${ }^{7}$ M. Bavia, F. Bertinelli, C. Taliani, and C. Zauli, Mol. Phys. 31, 479 (1976).

${ }^{8}$ C. D. Cooper, A. D. Williamson, J. C. Miller, and R. N. Compton, J. Chem. Phys. 73, 1527 (1980).

${ }^{9}$ J. L. Roebber, D. P. Gerrity, R. Hemley, and V. Vaida, Chem. Phys. Lett. 75, 104 (1980).

${ }^{10}$ L. Nyulaszi, J. Mol. Struct. 273, 133 (1992).

${ }^{11}$ M. B. Robin, Higher Excited States of Polyatomic Molecules (Acadamic, New York, 1975), Vol. II.

${ }^{12}$ G. L. Bendazoli, F. Bertineli, P. Palmieri, A. Brillante, and C. Taliani, J. Chem. Phys. 69, 5077 (1978).

${ }^{13}$ L. Nyilaszi and T. Veszpremi, J. Mol. Struct. 140, 253 (1986).

${ }^{14}$ L. Nyilaszi and T. Veszpremi, J. Mol. Struct. 140, 353 (1986).

${ }^{15}$ L. Nyilaszi and T. Veszpremi, Chem. Scr. 28, 331 (1988).

${ }^{16}$ R. Hakansson, B. Norden, and E. W. Thulstrup, Chem. Phys. Lett. 50, 305 (1977).

${ }^{17}$ B. Norden, R. Hakansson, P. B. Pedersen, and E. W. Thulstrup, Chem. Phys. 33, 355 (1978).

${ }^{18}$ N. Igarashi, A. Tajiri, and M. Hatano, Bull. Chem. Soc. Jpn. 54, 1511 (1981).
${ }^{19}$ W. M. Flicker, O. A. Mosher, and A. Kuppermann, J. Chem. Phys. 64, 1315 (1976).

${ }^{20}$ W. M. Flicker, O. A. Mosher, and A. Kuppermann, Chem. Phys. Lett. 38, 489 (1976).

${ }^{21}$ E. H. Van Veen, Chem. Phys. Lett. 41, 535 (1976).

${ }^{22}$ K. Tanaka, T. Nomura, T. Noro, H. Tatewaki, T. Takada, H. Kashiwagi, F. Sasaki, and K. Ohno, J. Chem. Phys. 67, 5738 (1977).

${ }^{23}$ W. Butscher and K.-H. Thunemann, Chem. Phys. Lett. 57, 224 (1978).

${ }^{24}$ K.-H. Thunemann, R. J. Buenker, and W. Butscher, J. Chem. Phys. 47, 313 (1980).

${ }^{25}$ D. C. Rawlings and E. R. Davidson, Chem. Phys. Lett. 98, 424 (1983).

${ }^{26}$ D. C. Rawlings, E. R. Davidson, and M. Gouterman, Int. J. Quantum Chem. 26, 237 (1984).

${ }^{27}$ H. Nakatsuji, O. Kitao, and T. Yonezawa, J. Chem. Phys. 83, 723 (1985).

${ }^{28}$ L. Serrano-Andres, M. Merchan, I. Nebot-Gil, B. O. Roos, and M. Fulscher, J. Am. Chem. Soc. 115, 6184 (1993).

${ }^{29}$ L. Serrano-Andres, M. Merchan, M. Fulscher, and B. O. Roos, Chem. Phys. Lett. 211, 125 (1993).

${ }^{30}$ M. H. Palmer, I. C. Walker, C. C. Ballard, and M. F. Guest, Chem. Phys. 192, 111 (1995).

${ }^{31}$ M. H. Palmer, I. C. Walker, and M. F. Guest, Chem. Phys. 238, 179 (1998).

${ }^{32}$ M. H. Palmer, I. C. Walker, and M. F. Guest, Chem. Phys. 241, 275 (1999).

${ }^{33}$ H. Nakano, T. Tsuneda, T. Hashimoto, and K. Hirao, J. Chem. Phys. 104, 2312 (1996).

${ }^{34}$ A. B. Trofimov and J. Schirmer, Chem. Phys. 214, 153 (1997).

${ }^{35}$ A. B. Trofimov and J. Schirmer, Chem. Phys. 224, 175 (1997).

${ }^{36}$ O. Christiansen and P. Jørgensen, J. Am. Chem. Soc. 120, 3423 (1998).

${ }^{37}$ O. Christiansen, J. Gauss, J. Stanton, and P. Jørgensen, J. Chem. Phys. 111, 525 (1999).

${ }^{38}$ J. Wan, M. Ehara, M. Hada, and H. Nakatsuji, J. Chem. Phys. 113, 5245 (2000).

${ }^{39}$ J. Wan, J. Meller, M. Hada, M. Ehara, and H. Nakatsuji, J. Chem. Phys. 113, 7853 (2000).

${ }^{40}$ J. H. Callomon, E. Hirota, K. Kuchitsu, W. J. Lafferty, A. G. Maki, and C. S. Pote, LANDOLT-BORNSTEIN, Numerical Data and Functional Relationships in Science and Technology, New Series, Group II: Atomic and Molecular Physics, Structure Data of Free Polyatomic Molecules (Springer-Verlag, Berlin-Heidelberg, 1976), Vol. 7.

${ }^{41}$ R. A. Kendall, T. H. Dunning, Jr., and R. J. Harrison, J. Chem. Phys. 96, 6796 (1992)

${ }^{42}$ T. H. Dunning, Jr., J. Chem. Phys. 90, 1007 (1989).

${ }^{43}$ K. Kaufmann, W. Baumeister, and M. Jungen, J. Phys. B 22, 2223 (1989).

${ }^{44}$ Gaussian 98, Revision A.1, M. J. Frisch, G. W. Trucks, H. B. Schlegel, et al., Gaussian, Inc., Pittsburgh, PA, 1998.

${ }^{45}$ H. Nakatsuji and K. Hirao, J. Chem. Phys. 68, 2053 (1978).

${ }^{46}$ H. Nakatsuji, Chem. Phys. Lett. 59, 362 (1978).

${ }^{47}$ H. Nakatsuji, Chem. Phys. Lett. 67, 329 (1979).

${ }^{48}$ H. Nakatsuji, Computational Chemistry: Reviews of Current Trends, edited by J. Leszczynski (World Scientific, Singapore, 1997), Vol. 2.

${ }^{49}$ H. Nakatsuji, Acta Chim. Hung. 129, 719 (1992).

${ }^{50}$ H. Nakatsuji, Chem. Phys. 75, 425 (1983).

${ }^{51}$ H. Nakatsuji, M. Hada, M. Ehara, J. Hasegawa, T. Nakajima, H. Nakai, O. Kitao, and K. Toyota, SAC/SAC-CI program system (SAC-CI96) for calculating ground, excited, ionized, and electron attached states and singlet to septet spin multiplicities. 